\title{
Simulating Chandra observations of galaxy clusters
}

\section{E. Rasia ${ }^{1}$, A. Gardini' ${ }^{2}$, P. Mazzotta ${ }^{3}$, G. Tormen ${ }^{1}$, S. De Grandi ${ }^{4}$ and L. Moscardini ${ }^{5}$}

\author{
${ }^{1}$ Dipartimento di Astronomia, Università di Padova, vicolo dell'Osservatorio 2, I-3512, Padova, \\ Italy email: rasia@pd.astro.it \\ ${ }^{2}$ Departement of Astronomy, University of Illinois, 1002 W. Green Street, Urbana, IL 61801, \\ USA \\ ${ }^{3}$ Dipartimento di Fisica, Università di Roma, via della Ricerca Scientifica, 1, Roma, Italy \\ ${ }^{4}$ INAF-Osservatorio Astronomico di Brera, via Bianchi 46, I-23807, Merate (LC), Italy \\ ${ }^{5}$ Dipartimento di Astronomia, Università di Bologna, via Ranzani 1, I-40127, Bologna, Italy
}

\begin{abstract}
The direct comparison of observations to numerical hydro-N-body simulations, although simple in principle, is not always trivial because of possible artificial effects produced by the instrument response and by instrumental and sky background. To overcome this problem we build the software package X-MAS (X-ray MAp Simulator) devoted to simulate X-ray observations of galaxy clusters obtained from hydro-N-body simulations.
\end{abstract}

\section{Introduction}

Theoretical studies of the dynamical processes underlying the formation and evolution of galaxy clusters are now mainly based on the results of numerical hydro-N-body simulations. Their results clearly show that clusters of galaxies experience violent events that release an enormous amount of energy in the intra-cluster medium (ICM). These events induce strong, but transient, variation of both ICM density and temperature, so that their distribution is far to be smooth and spherically symmetric. From the observational side, thanks to the superb angular and spectral resolution of the latest generation X-ray satellites, Chandra and XMM-Newton, we now know that many galaxy clusters, including the ones previously identified as relaxed, actually present a great deal of spatial features and have a rather complex thermal structure. Due to their complex nature and the impossibility of using simple de-projection techniques, most of these observed features can be quantitatively studied only through a direct comparison to numerical simulations. Ideally, to make these comparisons straightforward one needs to re-process the simulations themselves through a sort of virtual observatory that takes into account the instrumental response and the sky and the instrumental background.

\section{X-ray MAp Simulator: the method}

In this section we describe how the X-MAS package works. The package can be divided into two main units. The first unit is quite general and does not depend on the specific characteristics of the X-ray telescope. The second unit simulates the data relevant to an observation with a specific X-ray telescope and detector for a defined amount of time. At present our software package simulates Chandra observations in ACIS-S3 configuration only. The application of our simulation package to simulate Chandra in the ACIS-I mode and XMM-Newton observations requires an adaptation of this second unit only. In the 
following we summarize the steps of the two units, while a complete description is in Gardini et al. (2004).

First Unit: generating differential flux maps: The input of this unit is the output of a hydro-N-body simulation. For each gas particle in the simulation, we compute the emissivity and distribute it over the corresponding volume. After selecting a line of sight for the simulated observation, we compute the projected spectrum for each pixel and then the differential flux for each angular coordinate in bins of energy. The final step is adding Galactic absorption.

Second Unit: simulating Chandra observations: We estimate the expected number counts and iteratively subdivide the tile region until the counts become smaller than a given threshold; we then use the command FAKEIT in the utility XSPEC to convolve the spectral model of each subregion with the response of the ccd and to add the sky background; at the end we generate the final photon event file.

\section{ACIS-S3 observation of a simulated cluster}

We generate a $300 \mathrm{ks}$ ACIS-S3 Chandra observation of a high-resolution hydro-N-body simulation of a galaxy cluster presenting a major merger. It is selected from a sample of 17 clusters presented elsewhere (Rasia, Tormen \& Moscardini 2004; Tormen,Moscardini $\&$ Yoshida 2004). Since this particular simulation does not provide information on the cluster metallicity, we fixed its value to $Z=0.3 Z_{\odot}$ and we assumed a galactic equivalent column density of $N_{H}=5 \times 10^{20} \mathrm{~cm}^{-2}$.

The cluster flux map, obtained with the first unit of our X-MAS, is presented in Fig. 1 (left panel). The figure shows the flux in the $[0.1,10.0] \mathrm{keV}$ energy interval. The superimposed iso-contours are obtained from the image after a Gaussian smoothing with $\sigma=8^{\prime \prime}$. In the right panel of Fig. 1 we show the photon image of the $300 \mathrm{ks}$ Chandra ACIS-S3 observation of the simulated cluster, as obtained by applying the second unit of our software X-MAS. The image, extracted from the event file in the $[0.3,9.0] \mathrm{keV}$ energy band, is background-subtracted, vignetting-corrected, and binned to 1 arcsec. We notice that, as expected, after being observed with Chandra, the spatial features present in the simulation, but fainter than the instrument background, are no longer detected (for example, the three faint sub-clumps on the North, North-West and South-West, clearly visible). Conversely all the brighter features appear to be well reproduced by our simulator. We find that this is true even on scales as small as a few arcseconds.

\section{Spectral analysis: temperature maps}

In literature we usually find that the projected spectroscopic temperature $T_{\text {spec }}$ (obtained from the spectral analysis of the data), is directly compared with the so-called emission-weighted projected temperature $T_{e m}$ of the simulation. Unfortunately, in some cases $T_{\text {spec }}$ and $T_{e m}$ do not identify the same quantity. In fact, if the cluster has a complex thermal structure, the spectroscopic temperature is de-facto the result of the fit a "wrong" model (a single temperature model) to a multi temperature observed spectrum.

Using X-MAS we produced a Chandra observation of the simulated cluster and by performing a standard spectral analysis, we derived the spectroscopic temperature. This quantity is shown in the left panel of Fig. 2, while in the right panel we represent the emission-weighted temperature as derived directly from the simulation and computed using the same resolution of the spectroscopic temperature. If we now compare the two maps in Fig. 2, we notice that, although qualitatively similar, they show a number of important differences. Among others, we point our attention on the fact that: i) the 

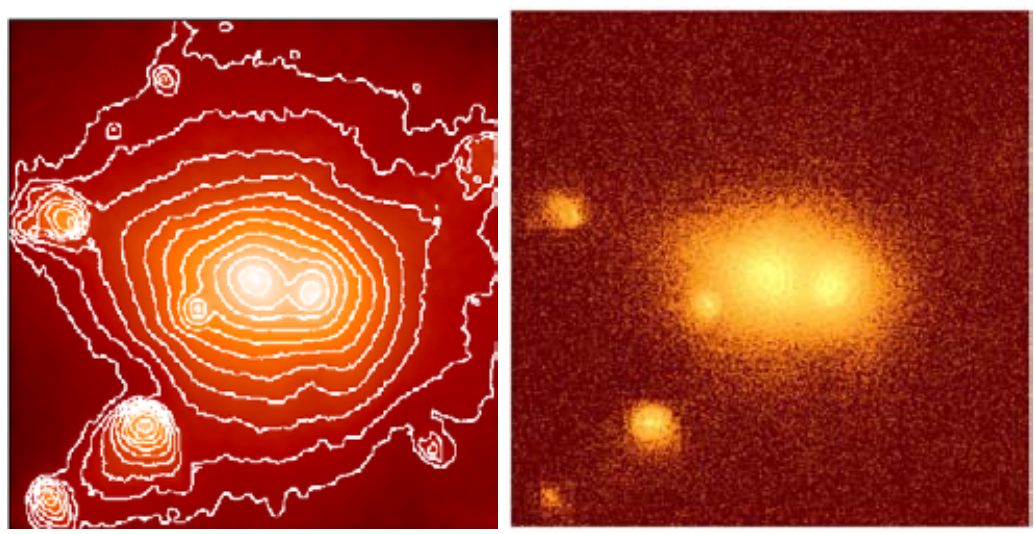

Figure 1. Left panel: Flux map of the simulated galaxy cluster, as obtained from the first unit. Right panel: photon image of the 300 ks ACIS-S3 observation.
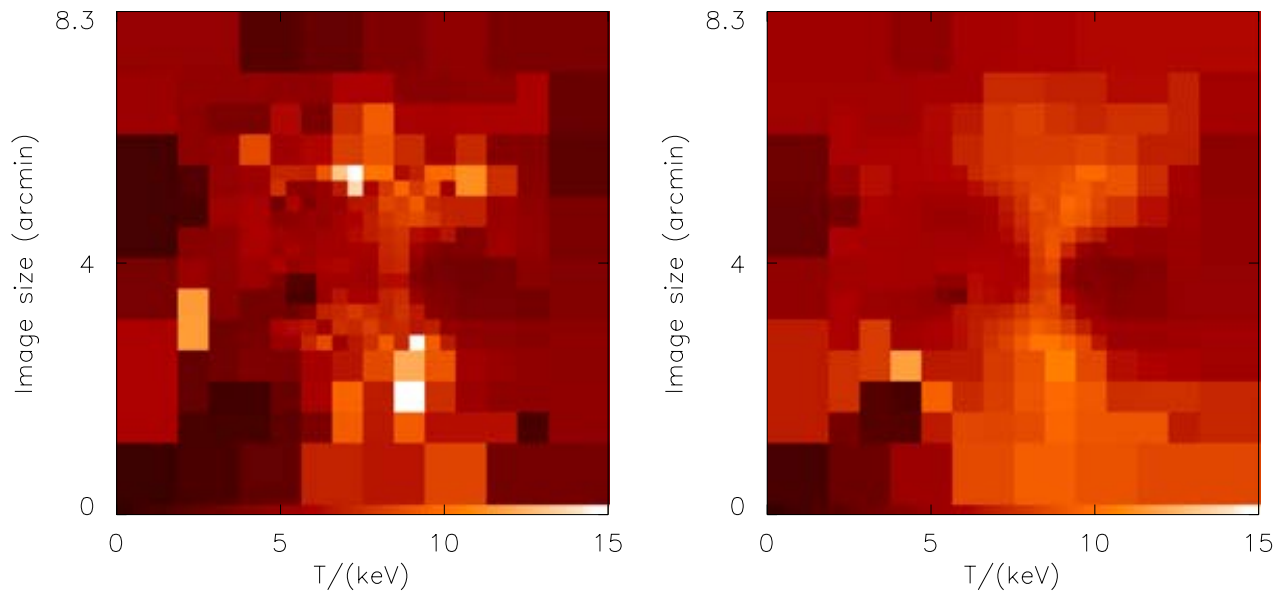

Figure 2. Temperature maps for the galaxy cluster. Left panel: map of the projected temperature, as derived from the spectroscopic analysis of the Chandra observation. Right panel: map of the emission-weighted temperature.

central merging blobs appear to be colder in the observed spectroscopic temperature map than in the $T_{e m}$; ii) the shock front produced by the motion of the innermost subclump in the lower-left corner, clearly visible in the emission-weighted map, is no longer detected in the spectroscopic temperature map.

To conclude we stress the fact that a proper comparison simulations-observations can only be done through the actual comparison of the spectral properties of both. This means that, in order to perform direct comparisons simulations-observations the simulated clusters must also be properly "observed" using X-ray observatory simulators like, for example, our X-MAS.

\section{References}

Gardini A., Rasia E., Mazzotta P., Tormen G., De Grandi S., Moscardini L. 2003, MNRAS in press, astro-ph/0310844

Rasia E., Tormen G., Moscardini L. 2004, MNRAS, 351, 237

Tormen G., Moscardini L., Yoshida N. 2004,MNRAS, 350, 1397 